\title{
Work Hour Rules and Contributors to Patient Care Mistakes: A Focus Group Study with Internal Medicine Residents
}

\author{
Kathlyn E. Fletcher, MD MA ${ }^{1,2}$ \\ Vikas Parekh, MD $^{3}$ \\ Lakshmi Halasyamani, MD ${ }^{4}$ \\ Samuel R. Kaufman, $\mathrm{MA}^{3}$ \\ Marilyn Schapira, MD, MPH ${ }^{1,2}$ \\ Kristyn Ertl, вA $^{1,2}$ \\ Sanjay Saint, MD, MPH ${ }^{5,3}$
}

${ }^{1}$ Clement J. Zablocki VA Medical Center, Milwaukee, Wisconsin

\footnotetext{
${ }^{2}$ Medical College of Wisconsin, Milwaukee, Wisconsin

${ }^{3}$ University of Michigan Medical School, Ann Arbor, Michigan

${ }^{4}$ St. Joseph Mercy Medical Center, Ann Arbor, Michigan

${ }^{5}$ Ann Arbor VA Medical Center, Ann Arbor, Michigan
}

BACKGROUND: The "Swiss cheese model" of systems accidents is commonly applied to patient safety, implying that many "holes" must align before an adverse event occurs. The Accreditation Council for Graduate Medical Education (ACGME) instituted work hour limitations to fill one such hole by reducing resident fatigue. OBJECTIVE: The objective of this study was to determine how residents perceive the impact of the ACGME rules and other factors on patient safety.

DESIGN: The study was designed as a focus group study.

PARTICIPANTS: Participating in the study were 28 internal medicine residents, of whom 13 were from a university-based program that includes both an academic medical center and a Veterans Affair (VA) hospital, 9 were from a communitybased program, and 6 were from a freestanding medical college that includes a large private teaching hospital and a VA hospital.

MEASUREMENT: Grounded theory analysis was used to examine transcripts of the focus group discussions.

RESULTS: A model of contributors to patient care errors emerged including fatigue, inexperience, sign-outs, not knowing patients, "entropy" (which we defined as "overall chaos in the system"), and workload. Participants described the impact of both intended and unintended consequences of the work hour rules on patient care. Residents reported improved well-being and less fatigue, but had concern about the effect of reduced continuity on patient care.

CONCLUSION: Our focus group participants perceived that the ACGME work hour limitations had minimized the impact of resident fatigue on patient care errors. Other contributors to errors remained and were often exacerbated by methods to maintain compliance with the rules. Journal of Hospital Medicine 2008;3: 228-237. (c) 2008 Society of Hospital Medicine.

KEYWORDS: patient safety, graduate medical education, physician staffing, qualitative research.

Datient safety can be understood in terms of the "Swiss cheese model" of systems accidents. This model implies that many "holes" must align before an adverse event occurs. ${ }^{1}$ The limitations on work hours instituted by the Accreditation Council for Graduate Medical Education (ACGME) ${ }^{2}$ sought to close one hole by reducing fatigue in residents. As programs comply with these regulations, new interventions are being implemented to limit resident hours. This has resulted in more handoffs of care and therefore less continuity. The ultimate result may be to increase patient care errors by opening up new holes, the opposite of the stated goal of this reform.

Some residency programs have reported on their experience 
TABLE 1

Call Structures on General Medicine Services of Sites Involved in Focus Groups

\begin{tabular}{|c|c|}
\hline Site & Call system on general medicine services \\
\hline Community & $\begin{array}{l}\text { - Four teams, each with } 1 \text { attending, } 1 \text { junior or senior resident, } 2 \text { interns. } \\
\text { - Teams take call every fourth day. Interns stay overnight and leave on the postcall day by } 1 \text { PM. Junior or senior resident on } \\
\text { team admits patients until } 9 \text { pM on call and returns at } 7 \text { AM postcall. Night float resident admits patients with on-call } \\
\text { interns from } 9 \text { PM until } 7 \mathrm{Am} \text {. } \\
\text { - On postcall day team resident stays the entire day, addressing all postcall clinical issues and follow-up. }\end{array}$ \\
\hline University & $\begin{array}{l}\text { At primary teaching hospital and VA: } \\
\text { - Four teams, each with } 1 \text { attending, } 1 \text { junior or senior resident, } 2 \text { interns. } \\
\text { - Teams take call every fourth day. Interns stay overnight, whereas residents leave at } 9 \text { PM on call and return at } 7 \text { AM postcall. } \\
\text { Night-float resident admits with interns from } 9 \text { PM to midnight, and then interns admit by themselves after midnight. } \\
\text { - Day-float resident present on postcall days to help team's senior resident finish the work. }\end{array}$ \\
\hline Freestanding medical college & $\begin{array}{l}\text { At primary teaching hospital: } \\
\text { - Six teams, each with } 1 \text { attending, } 1 \text { junior or senior resident, and } 1 \text { or } 2 \text { interns. } \\
\text { - Call is not as a team and is approximately every fifth day. Two residents and } 3 \text { interns take call overnight together. } \\
\text { At VA hospital: } \\
\text { - Four teams, each with } 1 \text { attending, } 1 \text { junior or senior resident, } 2 \text { interns. } \\
\text { - Teams take call every fourth day. One intern leaves at } 9 \text { PM on call and returns at } 7 \text { AM postcall; stays until } 4 \text { PM to cover team. }\end{array}$ \\
\hline
\end{tabular}

with hour reductions, giving insight into residents' perceptions on the benefits and drawbacks of such interventions. Residents have reported concern about continuity of care after such interventions. ${ }^{3-7}$ However, some residents believed they provided better patient care after the interventions to reduce hours. ${ }^{8,9}$ Few studies have actually documented changes in the incidence of adverse events or errors as a result of work hour limitations. ${ }^{10}$ One study conducted prior to implementation of the ACGME work hour rules demonstrated more complications in internal medicine patients after New York's Code 405 (a state regulation that limited resident work hours, similar to the ACGME rules) was implemented. ${ }^{11}$ In contrast, another study showed that errors committed by interns were reduced with scheduling changes that resulted in shorter shifts and reduced hours. ${ }^{12}$

Because residents are on the front lines of patient care, they are uniquely positioned to provide insight into the impact of the work hour rules on patient safety. We conducted this study to more fully understand the effect of the ACGME work hour limitations and other possible factors on patient care errors from the perspectives of internal medicine residents.

\section{METHODS}

\section{Participants and Sites}

All internal medicine residents and interns from 3 residency programs were recruited to participate in focus groups. We purposely chose programs based at diverse health care organizations. The first program was based at a university and had approximately 160 residents, who rotated at both the university hospital and the affiliated Veterans Affairs Medical Center (VAMC). The second program was based at a community teaching hospital and had approximately 65 residents. The third program was affiliated with a freestanding medical college and had approximately 95 residents, who rotated at a large, private tertiary-care hospital and also at the affiliated VAMC. Each program had a different call structure (Table 1).

Potential participants were recruited via E-mail, which explained that the study was about common scenarios for patient care errors and how the ACGME work hour rules affected patient care and errors.

\section{Design}

We conducted 4 focus groups in total (Appendix 1). The first 3 focus groups followed the same focus group guide, developed after a literature review. Focus groups 1 and 2 were conducted at the university-based program. Focus group 3 was conducted at the community teaching hospital-affiliated program. The first 3 focus groups were analyzed before the fourth focus group was conducted. A new focus group guide was developed for the fourth focus group to further explore themes identified in the first 3 focus groups (Fig. 1 and Appendix 2). The fourth focus group was conducted at the program affiliated with a freestanding medi- 
February 2004:

$1^{\text {st }}$ and $2^{\text {nd }}$ focus groups at university hospital

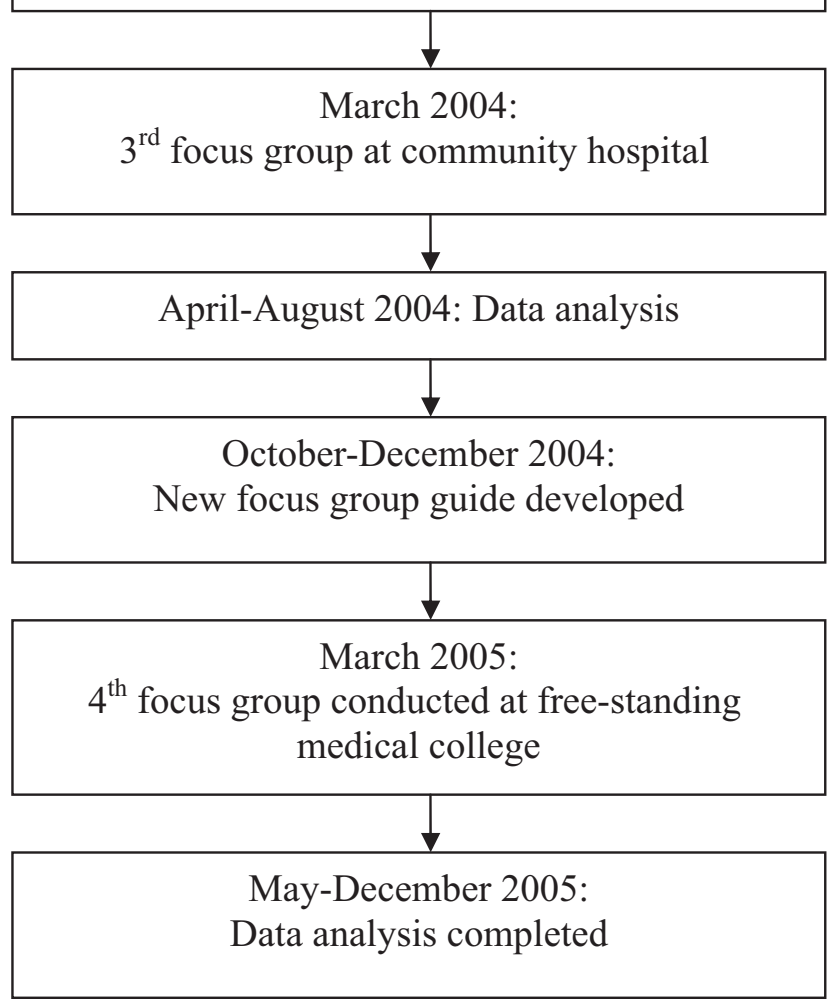

FIGURE 1. Time line of data collection and analysis.

cal college. All focus groups were audiotaped and transcribed verbatim. Each lasted approximately 90-120 minutes.

\section{Intervention}

The focus group guide for the first 3 focus groups consisted of main questions and follow-up prompts (Appendix 1). The focus group guide for the fourth focus group (Appendix 2) was developed based on themes from the first 3 focus groups, consistent with the iterative approach of grounded theory. ${ }^{13}$ Some of the questions were the same as in the first focus group guide; others were added to better understand the roles of faculty, teamwork, and inexperience in patient care errors.

Written informed consent was obtained before the focus groups began. Participants were paid $\$ 20$ and given dinner. All internal medicine residents at the institutions included were eligible. The focus groups were held after work. Each focus group comprised participants from a single institution.
The investigators who were the moderators were all junior faculty. They did not moderate the focus group at their own institution so as to minimize barriers to the residents' ability to speak freely about their experiences. The moderators prepared for their roles through discussion and assigned reading. ${ }^{14}$ The investigators used the focus group guide to ask questions of the group as a whole and facilitated the discussion that arose as a result. After each focus group, the moderator and assistant moderator debriefed each other about the important themes from the session.

\section{Ethics}

The institutional review boards at all sites approved this study.

\section{Analysis}

We used grounded theory to analyze the transcripts. ${ }^{15}$ Grounded theory is an iterative process that allows for themes to arise from the data. ${ }^{16}$ After the first 3 focus groups were completed, 5 of the investigators read all 3 transcripts at least twice and noted themes of interest in the text in a process of open coding. ${ }^{13}$ These investigators met in August 2004 to discuss the transcripts and the themes that had been identified by the individual investigators. A coding scheme of 33 codes was devised based on this meeting and the notes of individual investigators about the process of reading the transcripts. The need to conduct a fourth focus group to further explore certain issues was also identified. Two investigators (K.F., V.P.) independently coded the first 3 transcripts using the agreed-on coding scheme. One investigator used NVivo (QSR International, Doncaster, Australia), an appropriate software package, and the other investigator coded by hand. During this process, 2 additional themes were identified. The 2 coders agreed on the need to add them, and they were incorporated into the coding scheme, yielding a total of 35 codes. Three of the investigators met again to begin constructing a model to represent the relationships among the themes. The model was developed iteratively over the following year by considering the most important themes, their relationships to one another, unifying concepts identified during the textual analysis, and team meetings. To provide additional validity, peer checking occurred. Specifically, iterations of the model were discussed by the team of investigators, in local research-in-progress sessions, with groups of residents at 2 of the parti- 
TABLE 2

Demographic Characteristics of Study Participants

\begin{tabular}{ll} 
Number of participants by site & \\
Community & 9 \\
University & 13 \\
Freestanding medical college & 6 \\
Age (years), mean & 28.5 \\
Sex (female), $\mathrm{n}(\%)$ & $18(64 \%)$ \\
Postgraduate year, $\mathrm{n}(\%)$ & \\
Intern & $11(39 \%)$ \\
$\quad$ Second year and above & $17(61 \%)$ \\
Type of resident, $\mathrm{n}(\%)$ & \\
Categorical & $23(82 \%)$ \\
\hline
\end{tabular}

cipating institutions, and at national meetings. The fourth focus group was conducted at the third site in March 2005. The same 2 investigators applied the 35-code scheme and determined that thematic saturation had occurred; that is, no new themes were identified.

Agreement between the 2 coders was evaluated by reviewing $15 \%$ of each transcript and dividing the number of agreed-on codes by the total number of codes assigned to each section of text. The starting point of the text checked for agreement was chosen randomly. Agreement between the 2 coders for the first 3 focus groups was 43\%, 48\%, and 56\%, respectively. The fourth focus group was analyzed a year later, and the initial agreement between the coders was $23 \%$. After comparison and discussion, it was clear that 1 coder had coded many passages with more than 1 code, whereas the second coder had tried to choose the most pertinent code. The second coder recoded the transcript, and a new section was compared, resulting in agreement in $45 \%$ of that section. Discrepancies between the coders were resolved by consensus. None represented major differences of opinion; rather, they usually indicated the difficulty in choosing 1 primary code to fit an utterance that could be represented by several codes.

\section{RESULTS}

Twenty-eight residents participated. Some of these residents had experience in the pre-work hour era, and some did not. Average age was 28 years (range 26-33 years); 18 were women, and 11 were interns (Table 2). The focus groups ranged in size from 5 to 9. A sample of the codes and their definitions can be found in Table 3 .

\section{The Model}

The model (Fig. 2) illustrates resident-perceived contributors to patient care mistakes related to the ACGME work hour rules. These contributors are in the center circle. They include fatigue, inexperience, sign-out, not knowing their own patients well enough, "entropy" (which we defined as "the amount of chaos in the system"), and workload. They are not listed in order of importance. The boxes outside the circle are consequences of the ACGME work hour rules and their perceived impact on the contributors to patient care mistakes. At the top are the intended consequences, that is the specific goals of the ACGME: less resident time in the hospital (ie, reduced hours) and improved wellbeing. ${ }^{17}$ At the bottom are the unintended consequences: more patient care discontinuity and compliance with the work hour rules becoming a goal equally important to providing high-quality patient care. Of these 4 consequences, only improved wellbeing was viewed by the residents as decreasing patient care mistakes. The other consequences were cited by residents as sometimes increasing patient care errors. Because of the complexity of the model, several factors not directly related to resident work hours were identified in the analysis but are not shown in the model. They include faculty involvement and team work (usually positive influences), nurses and information technology (could be positive or negative), and late-night/early-morning hours (negative).

The quotations below illustrate the relationships between the consequences of the work hour rules, resident-perceived contributors to patient care mistakes, and actual patient care.

\section{Impact of Improved Well-Being}

Residents noted that improved well-being resulting from the work hour rules could mitigate the impact of fatigue on patient care, as described by this resident who discussed late-night admissions when on night float as opposed to on a regular call night. "...When I was night float, though, I was refreshed and more energized, and the patient...I think got better care because I wasn't as tired and...basically could function better. So I think that's a good part about this year is that I'm not as toxic, and I think I can think better...and care more when I'm not so tired, and my own needs have been met, in terms of sleep and rest and being home and stuff..."

Residents often described tension between the 
TABLE 3

Codes Contributing to the Model and Their Definitions

\begin{tabular}{|c|c|}
\hline Codes & Definitions \\
\hline Fatigue & $\begin{array}{l}\text { - How fatigue contributes to patient care problems. } \\
\text { - How not being fatigued contributes to improved patient care. }\end{array}$ \\
\hline Workload & $\begin{array}{l}\text { - How workload issues (eg, patient complexity) may contribute to patient care problems. } \\
\text { - Descriptions of times that workload was overwhelming: overextended-"Have to be in } 4 \text { places at once." }\end{array}$ \\
\hline Entropy & $\begin{array}{l}\text { - Residents' descriptions of too much of everything (information, interruptions); house of cards. } \\
\text { - How this chaos contributes to patient care problems. } \\
\text { - Being overwhelmed may be a facet. }\end{array}$ \\
\hline Not knowing own patients & $\begin{array}{l}\text { - Contributors to not knowing patients. } \\
\text { - How not knowing patients affects patient care. }\end{array}$ \\
\hline Sign-out/cross-cover & - Description of sign-out practices, problems, and solutions. \\
\hline Inexperience/lack of knowledge & $\begin{array}{l}\text { - How inexperience can contribute to patient care problems. } \\
\text { - Challenges and attributes of delivering patient care in the setting of learning to deliver patient care. }\end{array}$ \\
\hline Personal well-being & $\begin{array}{l}\text { - Discussions about residents lives, spouses, homes. } \\
\text { - How this affects patient care. }\end{array}$ \\
\hline Continuity of doctor care & $\begin{array}{l}\text { - Examples of discontinuity. } \\
\text { - How continuity and discontinuity contribute to patient care problems. } \\
\text { - Other aspects or attributes of continuity or discontinuity. }\end{array}$ \\
\hline Work hour rules as a goal & - Examples of compliance with ACGME rules becoming a goal in itself and its impact on patient care \\
\hline
\end{tabular}

benefits of being well rested and the benefits of continuity: "I don't know how it affects patient care unless you sort of make a leap and say that people who...have better well-being perform better. I don't know if that's true. Certainly, you could make the other argument and say if you're here all the time and miserable, and that's all you do, well, that's all you do. I'm not sure if maybe that's better. But I think for the physician when you compare them to lawyers...any other field, engineers, architects...I think they sort of have a more well-balanced life. So I think it is good for physician safety or their marriage safety. I'm not sure what it does with patient care."

\section{Impact of Having Less Time in the Hospital}

Having less time contributed to at least 2 factors, entropy and workload, as described in this passage: "I think with the...80-hour system there is a total of at least 1 less senior in house, if not more at times, and I know that when I was doing the night float thing and then even when I was doing senior call once, all it takes... is one sick patient that is too much for the intern alone to deal with,... and it's all of a sudden 6 in the morning, and there are 3 other admissions that the other intern has done that the senior hasn't seen yet, and that happened to me more than once." One resident discussed the workload on inpatient services: "I feel like I end up doing the same amount of work, but I have that much more pressure to do it all, and the notes are shorter, and you can't think through everything, and I actually find myself avoiding going in and talking to a family because I know that it is going to end up being a half-hour conversation when all I really wanted to do was to communicate what the plan was, but I don't have a chance to because I know it is going to turn into a longer conversation, and I know I don't have time to do that and get out on time."

\section{Impact of More Discontinuity}

Discontinuity could also exacerbate contributors to patient care mistakes, especially through sign-out/ cross-cover: "I think continuity of care is very important, obviously, whenever there is transition of caring for a patient from one physician to another physician...that information that gets transmitted 


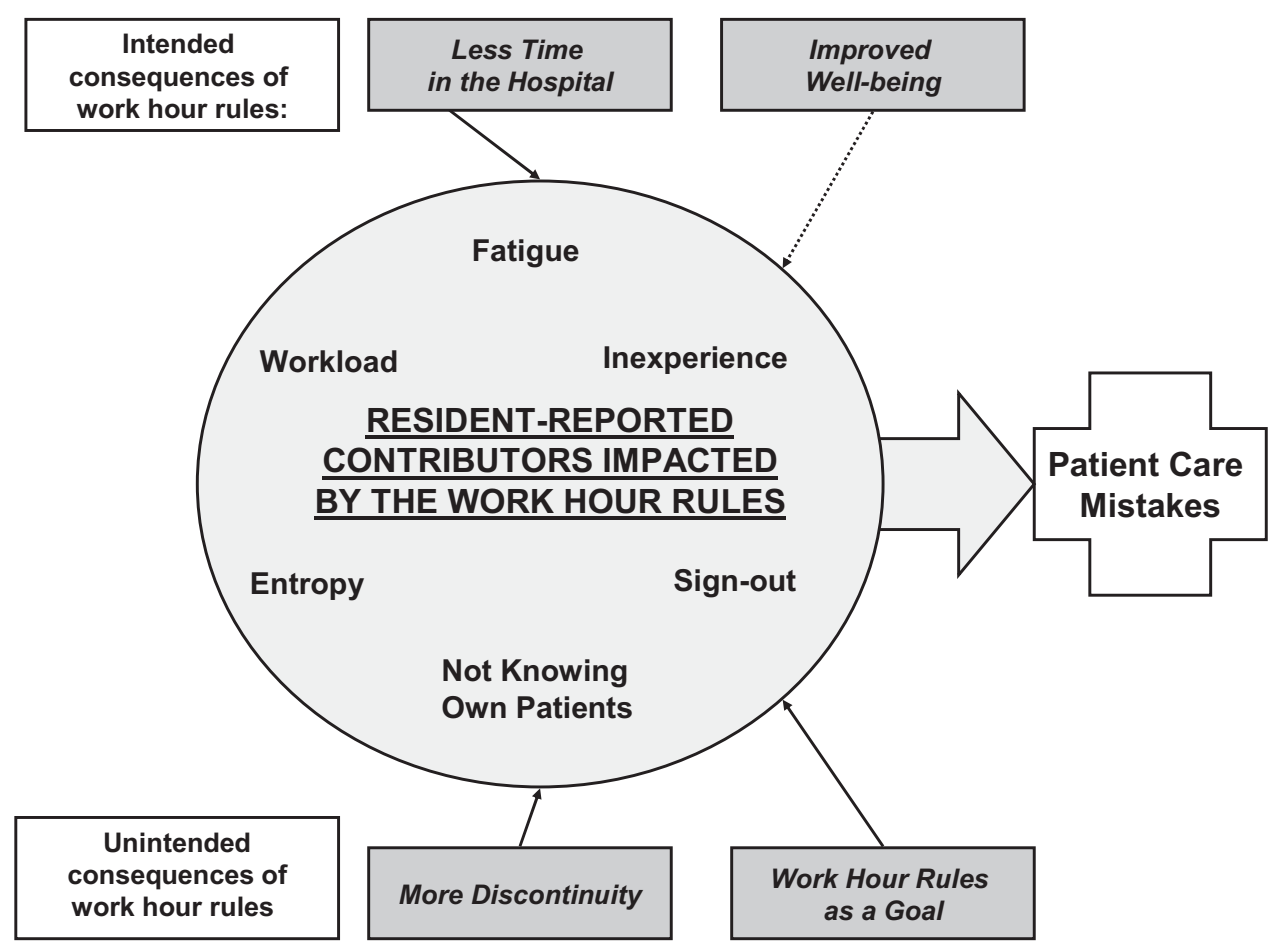

FIGURE 2. Conceptual model depicting how the ACGME work hour rules may affect resident-reported contributors to patient care mistakes. Solid arrows, aggravating factors; dotted arrows, mitigating factors.

from each other needs to be very well emphasized and clearly explained to the subsequent caretaker. And if that continuity of care is disrupted in some way, either through poor communication or lack of communication or a lot of different people having different responses to specific situations, that it can lead to [an] adverse event or medical errors like we just talked about."

Discontinuity also led to team members feeling they did not know their own patients well enough, which in turn could lead to mistakes in patient care. For example, residents described discharging patients on the wrong medications, overlooking important secondary problems, and failing to anticipate drug interactions. As a resident said: "I feel you almost have to [do] another $H$ and $P$ [history and physical] on the people that came in overnight, especially if they're going to be in the hospital some time because... the initial $H$ and $P$ and differentials oftentimes is going to change, and you have to be able to adjust to that.... I would say there's definitely errors there, coming on and making decisions without knowing the nuances of the history and physical.... So you essentially are making important decisions on patients you really don't know that well..." Another resident explained that the real problem with discontinuity was having inadequate time to get to know the patient: "The thing I always think about as far as continuity is...if you get a patient [transferred] to your care, how much time do you have which is allotted to you to get to know that patient? And actually, sometimes, I think that the continuity change in care is a good thing because you look at it through different eyes than the person before. So it really depends whether you have enough time to get to know them. On the other hand if you don't, then that's of course where errors I think occur."

Some also noted a sense of loss about not knowing their patients well: “...You have a sick patient at 1 o'clock, and...you have to turn their care over to your resident or the next intern who's on, and you know this patient best, they know you best, and you've got a relationship, and who knows? That patient might die in the next 12 hours, and you feel some sort of responsibility, but you're not allowed to stay and take care of them, and that kind of takes away a little bit of your autonomy and just like your spirit, I guess." 


\section{Impact of Having Compliance with Work Hour Rules Be a Goal}

Some residents reported problems when the work hour rules became the primary goal of team members. "I certainly have had some interns that I was supervising who made it clear that to them, the most important thing was getting out, and patient care maybe didn't even hit the list," explained one resident. "That bothers me a lot because I think that then that focus has become too strict, and the rules have become too important...I mean, if patient care has to happen for whatever reason-the patient's really sick-then there's enough flexibility to stay the half hour, hour; and I had an intern tell me that if she stayed the extra half hour that she would be over her 80 hours, and so she wasn't going to do it."

Having the rules as a goal affects the process of sign-out, as explained by a resident, “...because they want us to track time in and time out and are really strict about sticking particularly to the 30-hour portion of the rule, the 10 hours off between shifts, and I find that affecting patient care more than anything else because you feel like you can't stay that extra half an hour to wrap things up with a patient who you've been taking care of all night or to sit and talk with the family about something that came up overnight or...to do accurate and adequate documentation of things in order to hand that off to the next team because you got to get out of there..."

\section{DISCUSSION}

We conducted this study to better understand why internal medicine residents thought patient care mistakes occurred; we were particularly interested in how they perceived the impact of certain aspects of the ACGME work hour rules on patient care mistakes. Designing systems that achieve compliance with the work hour rules while minimizing patient risk can best be accomplished by fully understanding why errors occur.

Our study revealed that in the opinion of some interns and residents, the work hour rules had consequences for patient care. Like any intervention, this one had both intended and unintended consequences. ${ }^{18}$ The ACGME has stated that improvement in residents' quality of life was an intended consequence, ${ }^{17}$ and the participants in our study reported that this had occurred. Despite uncertainty about the overall impact on patient outcomes, residents were glad to have more time away from the hospital.
Our respondents reported that not knowing patients well was a factor that contributed to patient care errors. It is intuitive that working fewer hours often results in more handoffs of care, ${ }^{19}$ a situation characterized by not knowing patients well. However, residents also identified not getting to know their own patients well as a factor that led to patient care mistakes because of (1) incomplete knowledge of a patient's status, (2) delays in diagnosis, and (3) errors in management. They also described feelings of professional disappointment and frustration at not being able to perform certain aspects of patient care (eg, family meetings) because of the hour limits and the inflexibility of the rules. As we strive to redefine professionalism in the setting of reduced work hours, ${ }^{20}$ this phenomenon should be addressed.

Sign-out was identified as another contributor to patient care errors. The effectiveness of sign-outs is a concern across medicine, and the Joint Commission on Accreditation of Healthcare Organizations made sign-out procedures one of its priority areas in 2006. ${ }^{21}$ Much has been written about resident sign-out, emphasizing the relationship between poor-quality sign-outs and patient safety. ${ }^{19,22}$ However, barriers to effective sign-out processes persist, ${ }^{23}$ even though standardized signout strategies have been described. ${ }^{24,25}$ Even in a rigorous study of work hours and patient safety, the computerized sign-out template for the residents was rarely used. ${ }^{12}$ Cross-coverage, or the patient care that occurs after sign-out is complete, has also been linked to a greater likelihood of adverse events. ${ }^{26}$

Several factors not related to resident work hours were noted to often mitigate patient care mistakes. Physician teamwork, nursing, information technology (eg, computerized medical records), and faculty supervision were the most prominent. For example, the information technology available at the VA hospitals often helped to facilitate patient care, but it also provided an overwhelming amount of information to sift through. It was clear that the influence of some of these factors varied from institution to institution, reflecting the cultures of different programs.

Our results are consistent with those reported from previous studies. Striking a balance between preventing resident fatigue and preserving continuity of care has been debated since the ACGME announced changes to resident work hour limits. ${ }^{27}$ Resident quality of life generally improves and fa- 
tigue decreases with work hour limits in place, ${ }^{28}$ but patient safety remains a concern. ${ }^{10}$ Our findings corroborate the benefits of improved resident wellbeing and the persistent concerns about patient safety, identified in a recently published study at a different institution. ${ }^{29}$ However, our findings expand on those reported in the literature by offering additional empirical evidence, albeit qualitative, about the way that residents see the relationships among the consequences of work hour rules, resident-reported contributors to patient care mistakes, and the mistakes themselves.

Our study should be interpreted in the context of several limitations. First, the use of qualitative methods did not allow us to generalize or quantify our findings. However, we purposely included 3 diverse institutions with differing responses to the work hour rules to enhance the external validity of our findings. Second, the last focus group was conducted a year after the first 3 ; by that point, the work hour rules had been in place for 20 months. We believe that this was both a strength and a limitation because it allowed us to gain a perspective after some of the initial "growing pains" were over. This time lag also allowed for analysis of the first 3 transcripts so we could revise the focus group guide and ultimately determine that thematic saturation had occurred. In addition, few of our questions were phrased to evaluate the ACGME rules; instead, they asked about links among discontinuity, scheduling, fatigue, and patient care. We therefore believe that even residents who were not in the programs before the work hour rules began were still able to knowledgeably participate in the conversation. One question directly referable to the ACGME rules asked residents to reflect on problems arising from them. This could have led residents to only reflect on the problems associated with the rules. However, in all 4 focus groups, residents commented on the positive impact of improved well-being resulting from the work hour rules. This led us to believe the respondents felt they could voice their favorable feelings as well as their unfavorable feelings about the rules. An additional limitation is that the agreement between coders was only $45 \%$. It is important to realize that assessing coding agreement in qualitative work is quite difficult because it is often difficult to assign a single code to a section of text. When the coders discussed a disagreement, it was almost always the case that the difference was subtle and that the coding of either investigator would made sense for that text.
Finally, our results are based on the participation of 28 residents. To be certain we were not representing the opinions of only a few people, we presented iterations of this model to faculty and resident groups for their feedback. Importantly, the residents offered no substantial changes or criticisms of the model.

Limitations notwithstanding, we believe our findings have important policy implications. First, despite work hours successfully being reduced, residents perceived no decrease in the amount of work they did. This resulted in higher workload and more entropy, suggesting that residency programs may need to carefully evaluate the patient care responsibility carried by residents. Second, continued effort to educate residents to provide effective signout is needed. As one participant pointed out, residency offers a unique opportunity to learn to manage discontinuity in a controlled setting. Another educational opportunity is the chance to teach physician teamwork. Participants believed that effective teamwork could ameliorate some of the discontinuity in patient care. This teamwork training should include faculty as well, although further work is needed to define how faculty can best add to patient continuity while still fostering resident autonomy. Finally, the impact of work hour rules on the professional development of residents should be further explored.

In conclusion, we have proposed a model to explain the major resident-reported contributors to patient care mistakes with respect to resident work hour rules. Our results help to clarify the next steps needed: testing the proposed relationships between the factors and patient care mistakes and rigorously evaluating solutions that minimize the impact of these factors. Returning to the Swiss cheese framework for describing systems accidents, our results suggest that although resident work hour reductions may have sufficiently filled the hole caused by resident fatigue, other gaps may have actually widened as a result of the systems put into place to achieve compliance. Continued vigilance is therefore necessary to both identify the additional holes likely to appear and, more importantly, effectively close those holes before patient harm occurs.

\section{APPENDIX 1. INITIAL FOCUS GROUP GUIDE (FOCUS GROUPS 1-3)}

1. How would you define the following: a. A medical error? 
b. An adverse patient event?

The IOM definition of a medical error is "the failure of a planned action to be completed as intended or the use of a wrong plan to achieve an aim" (IOM report summary). From this point on, let us try to use this definition when we refer to errors.

2. What is the impact of continuity of care on medical errors, mistakes, or adverse outcomes?

a. Team versus individual continuity.

3. What are some settings at the hospitals where you work in which you have seen mistakes, errors, or bad outcomes in patient care?
a. Time of day?
b. Day in call cycle?
c. Other factors?

4. What types of mistakes, errors, or bad outcomes do you notice with patient care at the hospitals where you work? Please describe.

5. What are the things that contribute to patient-related mistakes, errors, or bad outcomes at the hospitals where you work? (If needed, some prompts include)

a. How does fatigue contribute?

b. How do days off or lack of days off contribute?

c. What are the effects of nurses?

6. What types of mistakes, errors, or bad outcomes have you noticed with transitions in care (eg, signouts, cross-coverage) in your patients at the hospitals where you work? Please describe.

7. How has technology impacted errors, mistakes, and adverse outcomes?
a. PDA.
b. Computer access.
c. Computer-order entry (if applicable).

8. What problems have you seen with the new ACGME regulations on work hours at the hospitals where you work?

9. What are some possible solutions?

\section{APPENDIX 2. FOCUS GROUP GUIDE (4TH FOCUS GROUP)}

The IOM definition of a medical error is " the failure of a planned action to be completed as intended or the use of a wrong plan to achieve an aim."

1. Please describe the call structure at each institution where you do ward months (eg, non-ICU months).

2. What are some settings at the hospitals where you work where you have seen medical errors, mistakes, or adverse outcomes?

3. How do you think that other nurses influence the occurrence of medical errors, mistakes, or adverse outcomes?
a. Clerks?
b. Other ancillary staff?

4. How would you describe the responsibilities of a cross-covering resident or intern?

5. How do you think continuity of care impacts patient care in terms of medical errors, mistakes, or adverse outcomes? a. What role do sign-outs have?

6. How do you think that fatigue impacts patient care in terms of medical errors, mistakes, or adverse outcomes?

7. How do you think that technology such as computerized physician order entry impacts patient care in terms of medical errors, mistakes, or adverse outcomes?

a. Electronic medical records?

b. Palm pilots?

c. Is there such a thing as too much information?

8. How do you think that experience (or inexperience) impacts patient care in terms of medical errors, mistakes, or adverse outcomes?

9. Please describe how attendings supervise you when you are on a ward team. How do you think that attending supervision impacts patient care in terms of medical errors, mistakes, or adverse outcomes?

a. What about resident supervision of interns?

b. What is the ideal role of an attending on a team?

c. Can you think of a time when having attending input changed the plans or the course of a patient in a major way, good, bad, or neutral?

10. How do you think that time of day impacts patient care in terms of in terms of medical errors, mistakes, or adverse outcomes?

a. How comfortable do you feel calling for help at night? What makes you more or less likely to do it (personal attributes of person to be called, situation, etc.)?

11. What do you think is an ideal workload? (eg, How many complex patients are typical of your hospitals?) Does that vary from the VA to St. Joe's to Froedtert? How many patients should be admitted in 1 night by an intern? How many should an intern have ongoing responsibility for? Is there such a thing as too few patients?

12. If one of your family members were to admitted to your hospital at night with a life-threatening 
condition, which situation would you prefer for their care (all other things being equal): admission by night float with handoff to a new but well-rested resident or admission by a resident who then continues to care for that family member the next day but has been awake for 24 hours, admitting and cross-covering other patients? Why?

13. What do you think was the intent of the ACGME rules? Do you think that those goals have been accomplished? Why or why not? How have they affected you as residents? How do you think that the ACGME work hour rules have influenced patient care?

Address for correspondence and reprint requests: Kathlyn E. Fletcher, MD, MA, Primary Care Division, Clement J. Zablocki VAMC, 5000 W. National Avenue, Milwaukee, WI 53295; Fax: (414) 382-5017; E-mail: kfletche@mcw.edu

Received 6 December 2006; revision received 28 August 2007; accepted 1 September 2007.

\section{REFERENCES}

1. Reason J. Human error: Models and management. Br Med J. 2000;320:768-770.

2. Philibert I, Friedmann P, Williams WT, ACGME Work Group on Resident Duty Hours, Accreditation Council for Graduate Medical Education. New requirements for resident duty hours. JAMA. 2002;288:1112-1114.

3. Kelly A, Marks F, Westhoff C, Rosen M. The effect of the New York State restrictions on resident work hours. Obstet Gynecol. 1991;78(3 Pt 1):468-473.

4. Trontell MC, Carson JL, Taragin MI, Duff A. Impact of a night float system on internal medicine residency programs. Acad Med. 1991;66:370.

5. Mather HM. Coping with pressures in acute medicine. The Royal College of Physicians Consultant Questionnaire Survey. J R Coll Physicians Lond. 1998;32:211-218.

6. Daigler GE, Welliver RC, Stapleton FB. New York regulation of residents' working conditions. 1 year's experience. Am J Dis Child. 1990;144:799-802.

7. Baldwin PJ, Newton RW, Buckley G, Roberts MA, Dodd M. Senior house officers in medicine: Postal survey of training and work experience. $\mathrm{Br}$ Med J. 1997;314:740-743.

8. Druss BG, Pelton G, Lyons L, Sledge WH. Resident and faculty evaluations of a psychiatry night-float system. Acad Psychiatry. 1996;20(1):26-34.

9. Yedidia MJ, Lipkin M, Jr., Schwartz MD, Hirschkorn C. Doctors as workers: work-hour regulations and interns' perceptions of responsibility, quality of care, and training. J Gen Intern Med. 1993;8:429-435.

10. Fletcher KE, Davis SQ, Underwood W, Mangrulkar RS, McMahon LF Jr, Saint S. Systematic review: effects of resident work hours on patient safety [review] [39 refs]. Ann Intern Med. 2004;141:851-857.
11. Laine C, Goldman L, Soukup JR, Hayes JG. The impact of a regulation restricting medical house staff working hours on the quality of patient care. JAMA. 1993;269:374-378.

12. Landrigan CP, Rothschild JM, Cronin JW, et al. Effect of reducing interns' work hours on serious medical errors in intensive care units [see comment]. N Engl J Med. 2004;351: 1838-1848.

13. Creswell JW. Qualitative Inquiry and Research Design: Choosing among Five Traditions. Thousand Oaks, CA: Sage Publications, Inc.; 1998.

14. Krueger RA. Moderating Focus Groups. Thousand Oaks, CA: Sage Publications; 1998.

15. Glaser BG, Strauss AL. The Discovery of Grounded Theory: Strategies for Qualitative Research. Chicago, IL: Aldine Publishing Company; 1967.

16. Strauss A, Corbin J. Basics of Qualitative Research: Techniques and Procedures for Developing Grounded Theory. Vol. 2. Thousand Oaks, CA: Sage Publications; 1998.

17. ACGME. Statement of Justification/Impact for the Final Approval of Common Standards Related to Resident Duty Hours. Available at: http://www.acgme.org/DutyHours/impactStatement.pdf. Accessed February 21, 2003.

18. Worthen BRS, J. R. Fitzpatrick J. L. Program Evaluation: Alternative Approaches and Practical Guidelines. New York, NY: Longman; 1997.

19. Vidyarthi A. Fumbled handoff. Web M\&M (Morbidity and Mortality Rounds on the Web. Available at: http://www. webmm.ahrq.gov/printview.aspx? caseID $=55$. Accessed December 27, 2005.

20. Rosenbaum JR. Can residents be professional in 80 or fewer hours a week? Am J Med. 2004;117:846-850.

21. Helpful solutions for meeting the 2006 national patient safety goals. Jt Comm Perspect Patient Saf. 2005;5(8):1-20.

22. Gandhi TK. Fumbled handoffs: one dropped ball after another. Ann Intern Med. 2005;142:352-358.

23. Solet DJ, Norvell JM, Rutan GH, Frankel RM. Lost in translation: challenges and opportunities in physician-to-physician communication during patient handoffs. Acad Med. 2005;80:1094-1099.

24. Simpson KR. Handling handoffs safely. Am J Matern Child Nurs. 2005;30(2):152.

25. Patterson ES, Roth EM, Woods DD, Chow R, Gomes JO. Handoff strategies in settings with high consequences for failure: lessons for health care operations. Int J Qual Health Care. 2004;16(2):125-132.

26. Petersen LA, Brennan TA, O'Neil AC, Cook EF, Lee TH. Does housestaff discontinuity of care increase the risk for preventable adverse events? Ann Intern Med. 1994;121:866-872.

27. Fletcher KE, Saint S, Mangrulkar RS. Balancing continuity of care with residents' limited work hours: defining the implications. Acad Med. 2005;80(1):39-43.

28. Fletcher KE, Underwood W, Davis SQ, Mangrulkar RS, McMahon LF, Saint S. Effects of work hour reduction on residents' lives: a systematic review. JAMA. 2005;294:1088-1100.

29. Lin GA, Beck DC, Garbutt JM. Residents' perceptions of the effects of work hour limitations at a large teaching hospital. Acad Med. 2006;81(1):63-67. 